\title{
Assessing genotype-phenotype correlation in Costello syndrome using a severity score
}

\author{
Elizabeth M. McCormick, MS1', Elizabeth Hopkins, MS'1, Laura Conway, PhD², Sarah Catalano, BS², \\ Jobayer Hossain, $\mathrm{PhD}^{4}$, Katia Sol-Church, PhD ${ }^{4}$, Deborah L. Stabley, BS ${ }^{4}$ and Karen W. Gripp, MD ${ }^{1}$
}

Purpose: Costello syndrome, a rare genetic disorder with multisystemic involvement, is caused by germline HRAS mutations. Because several different missense mutations have been reported, a severity scoring system was developed to assess a possible genotype-phenotype correlation.

Methods: Records of 78 individuals with Costello syndrome were scored in early childhood, childhood, and young adulthood by a reviewer blinded to the individuals' specific mutations. These scores were based on certain medically relevant feeding, neurologic, orthopedic, endocrine, cardiac, malignancy, and mortality manifestations. Individuals' severity scores were then grouped by the particular HRAS mutation. The mixed-model approach for repeated-measures analysis of variance with unstructured within-subject correlation, pairwise comparisons, and contrast were used to determine whether the severity scores differed by mutation.
Results: Although the sample size was small, individuals with the p.G12A or p.G12C HRAS change were more severely affected than those with other HRAS mutations. Regardless of the mutation, severity did not increase significantly over time.

Conclusion: Despite its limitations, including the small number of individuals with rare mutations and possibly incomplete medical records, this work providing the first quantitative assessment of phenotypic severity in a Costello syndrome cohort supports a medically relevant genotype-phenotype correlation.

Genet Med 2013:15(7):554-557

Key Words: Costello syndrome; genotype-phenotype correlation; HRAS; severity score

\section{INTRODUCTION}

Costello syndrome (OMIM no. 218040) is a rare genetic disorder characterized by failure-to-thrive in infancy, short stature, characteristic facial features, curly or sparse hair, papillomata, osteoporosis, cardiovascular malformations such as pulmonic stenosis and hypertrophic cardiomyopathy, and rhythm disturbances such as multifocal atrial tachycardia, ${ }^{1}$ neurological abnormalities including intellectual disability, ${ }^{2}$ syringomyelia, Chiari I malformation, and hydrocephalus; ${ }^{3}$ and a predisposition to malignancies. ${ }^{4}$ Costello syndrome is caused by heterozygous germline HRAS mutations that typically occur de novo. . $^{5-9}$ Although more than $80 \%$ of patients share the p.G12S HRAS mutation, other missense mutations have been reported. ${ }^{10}$

Genotype-phenotype correlations have been characterized for several HRAS mutations, with associations found between the p.G12A HRAS change and increased predisposition to malignancy, ${ }^{8}$ p.G12C and p.G12D and severe hypertrophic cardiomyopathy and neonatal mortality, ${ }^{11}$ p.T58I and p.A146V and milder facial findings, ${ }^{12}$ and p.G13C and lower rates of neurological abnormalities requiring surgery, lack of multifocal atrial tachycardia and papillomata, and long eyelashes requiring trimming. ${ }^{13}$ Here, we describe the implementation of a severity scoring system in order to standardize and quantify genotypephenotype correlation of medically relevant manifestations in Costello syndrome.

\section{MATERIALS AND METHODS}

\section{Participants}

The 78 individuals in this study were enrolled in an institutional review board-approved research study (IRB Nemours Delaware 2005-051) and have undergone molecular analysis to determine their specific HRAS change (Table 1). The participants ranged in age from 1 to 42 years. Their reviewed records contained information obtained through parental interview and were supported by medical documentation whenever available.

\section{Severity score}

A severity score was developed to quantitatively analyze the severity of Costello syndrome. Findings were chosen on the basis of their medical relevance, excluding characteristics such as curly hair, long eyelashes, or deep palmar creases. Lack of sufficiently reliable and comparable measurements characterizing developmental delay or intellectual disability precluded using these doubtlessly very relevant problems in our scoring system. Points were given for each characteristic being scored (Table 2). Most characteristics present scored a single point. The presence of a malignancy scored two points, as did an additional primary malignancy, with no points for recurrences. In addition to Achilles tendon lengthening, the first orthopedic surgery scored one point; however, additional orthopedic

${ }^{1}$ Division of Medical Genetics, Alfred I. duPont Hospital for Children, Wilmington, Delaware, USA; ${ }^{2}$ Genetic Counseling Program, Arcadia University, Glenside, Pennsylvania, USA; ${ }^{3}$ Tufts University School of Medicine, Boston, Massachusetts, USA; ${ }^{4}$ Department of Biomedical Research, Nemours' Children’s Clinic, Wilmington, Delaware, USA. Correspondence: Karen W. Gripp (kgripp@nemours.org) 


\begin{tabular}{lcc}
$\begin{array}{l}\text { Table } 1 \text { HRAS mutation groups } \\
\text { HRAS mutation } \\
\text { present }\end{array}$ & $\begin{array}{c}\text { Number of } \\
\text { individuals }\end{array}$ & $\begin{array}{c}\text { Age range of individuals, } \\
\text { mean (range) in years }\end{array}$ \\
\hline p.G12S & 60 & $14(1-42)$ \\
p.G12A & 4 & $11(2-29)$ \\
p.G12C & 3 & $2(1-4)$ \\
p.G13C & 7 & $8(1-19)$ \\
p.T58I & 2 & $7(4-9)$ \\
p.A146V & 1 & 8 \\
p.G13D & 1 & 22 \\
Total & 78 & $13(1-42)$
\end{tabular}

Table 2 Costello syndrome severity scoring system

\begin{tabular}{|c|c|}
\hline Characteristics & $\begin{array}{l}\text { Points } \\
\text { scored }\end{array}$ \\
\hline \multicolumn{2}{|l|}{ Feeding difficulty } \\
\hline Feeding tube required & 1 \\
\hline \multicolumn{2}{|l|}{ Cardiac abnormalities } \\
\hline Arrhythmia & 1 \\
\hline Ectopic atrial tachycardia/multifocal atrial tachycardia & 1 \\
\hline Hypertrophic cardiomyopathy (HCM) & 1 \\
\hline Surgery for HCM & 1 \\
\hline \multicolumn{2}{|l|}{ Orthopedic abnormalities } \\
\hline Achilles tendon lengthening surgery & 1 \\
\hline Orthopedic surgery, other & 1 \\
\hline \multicolumn{2}{|l|}{ Neurological abnormalities } \\
\hline Chiari I malformation requiring surgery & 1 \\
\hline Hydrocephalus requiring surgery & 1 \\
\hline Seizures requiring medication & 1 \\
\hline \multicolumn{2}{|l|}{ Malignancies } \\
\hline Primary occurrence & 2 \\
\hline Additional primary & 2 \\
\hline \multicolumn{2}{|l|}{ Bone density } \\
\hline$Z$ score $<-3$ or bisphosphonate infusion & 1 \\
\hline \multicolumn{2}{|l|}{ Short stature } \\
\hline Height $<-3$ SD or growth hormone use & 1 \\
\hline \multicolumn{2}{|l|}{ Mortality } \\
\hline Before the age of 1 year & 8 \\
\hline After the age of 1 year & 4 \\
\hline
\end{tabular}

surgeries did not score in order to prevent inflated scores from multiple revisions.

A severity score was assessed for each individual at the age of 2 years (early childhood score), 10 years (childhood score), and finally at 20 years (young adulthood score) to assess the severity over time. Scores from the previous age interval were carried over to each subsequent age interval, preventing a decrease over time. Although severity was assessed at three ages listed above, the only age at which all individuals studied were represented was early childhood because some individuals were still in early childhood and others had died before the age of 20 years.

After all the individuals had been scored, those with the same HRAS mutation were grouped. The early childhood, childhood, and young adulthood scores of each group were then compared with the other HRAS mutation groups.

\section{Data analysis}

Mixed-model repeated-measures analysis of variance with unstructured within-subject correlation was used to compare mean scores between mutation groups and within-subject changes over time. Pairwise comparisons and contrast were used to perform specific comparisons of interest. Severity of Costello syndrome over time was analyzed to see if severity became significantly worse over the course of an individual's life, or if there are any specific time periods where the severity of Costello syndrome worsens significantly. All tests were two tailed with a level of significance of $<0.05$.

\section{RESULTS}

\section{Costello syndrome severity over time}

Costello syndrome, as based on the associated medical issues scored, was not found to significantly worsen between the ages of 2 and 20 years (Supplementary Figure S1 online; Supplementary Table S1 online).

\section{Mortality}

Five of the 78 individuals in this study died: one in adulthood, one in childhood, and three in early childhood, including one in the first year of life. One had the mutation predicting p.G12C, one had the mutation predicting p.G12A, and three had the mutation predicting p.G12S. Two individuals scored two points before their death, one individual scored three points, and two individuals scored five points (excluding points given for mortality). These two individuals died between the age of 1 and 2 years and had acquired these points during early childhood. Of note, all five deceased individuals scored points for requiring a feeding tube, four of five for arrhythmia (with one scoring an additional point for multifocal atrial tachycardia/ectopic atrial tachycardia), three of five for hypertrophic cardiomyopathy, and two of five for a malignancy.

\section{Early childhood severity}

The p.G12A and p.G12C mutation groups had the two highest average severity scores in early childhood and childhood. This was different for the young adulthood severity score because no individuals with p.G12C had reached this age (Table 3). The analysis of our data focused on the early childhood age because each individual studied was represented. The p.G12S mutation group was used for comparison (Table 4) because it is found in the majority of individuals with Costello syndrome. The mean differences between those with p.G12A and p.G12S, and between those with p.G12C and p.G12S, were considerably high as compared with the standard error. Therefore, a significant difference was found comparing the mean of the early childhood severity scores of p.G12S individuals with the means of the early childhood scores of individuals with p.G12A $(P=0.05)$ and p.G12C $(P=0.02)$.

Higher severity scores for individuals with the p.G12A or p.G12C HRAS mutation were also seen upon combining these 
Table 3 Mean severity scores (SE) for each mutation group at each age

Mean severity scores

\begin{tabular}{lc}
\hline${\text { Early childhood score }(2 \text { years })^{\mathrm{a}}}$ & \\
p.G12S & $2.017(0.257)$ \\
p.G12A & $4.500(0.997)$ \\
p.G12C & $5.333(1.151)$ \\
p.G13C & $1.857(0.754)$ \\
p.T58I & $0.500(1.410)$ \\
Childhood score (10 years) ${ }^{\mathrm{a}}$ & \\
p.G12S & $3.743(0.310)$ \\
p.G12A & $6.016(1.271)$ \\
p.G12C & $5.098(1.872)$ \\
p.G13C & $2.188(0.930)$ \\
p.T58I & $1.000(1.680)$ \\
Young adulthood cumulative score (20 years) & \\
p.G12S & $4.274(0.315)$ \\
p.G12A & $6.552(1.343)$ \\
p.G12C & $-{ }^{\mathrm{b}}$ \\
p.G13C & $1.977(1.202)$ \\
p.T58I & $-{ }^{\mathrm{b}}$ \\
\hline
\end{tabular}

andividuals with p.A146V and p.G13D are excluded because only one individual each had these mutations. 'The individuals with these mutations have not yet reached this age.

Table 4 Pairwise comparison of most common Costello syndrome-causing mutation (p.G12S) to other mutations in early childhood

\begin{tabular}{lccc}
\multicolumn{2}{l}{ Mutations compared $^{\text {a }}$} & Mean difference (SE) & $\boldsymbol{P}$ value \\
\hline p.G12S & p.G12A & $2.48(1.23)$ & $\mathbf{0 . 0 5}$ \\
p.G12S & p.G12C & $3.32(1.37)$ & $\mathbf{0 . 0 2}$ \\
p.G12S & p.G13C & $0.16(0.93)$ & 0.87 \\
p.G12S & p.T58I & $1.52(1.57)$ & 0.36 \\
\hline
\end{tabular}

$P$ values shown in bold are statistically significant.

andividuals with p.A146V and p.G13D are excluded because only one individual each had these mutations.

Table 5 Comparison of mutation groups at early childhood

\begin{tabular}{|c|c|c|c|}
\hline \multicolumn{2}{|c|}{ Mutation groups compared } & \multirow{2}{*}{$\begin{array}{c}\text { Difference (SE) } \\
2.84(1.02)\end{array}$} & \multirow{2}{*}{$\begin{array}{c}P \text { value } \\
0.009\end{array}$} \\
\hline p.G12S & p.G12A, p.G12C & & \\
\hline p.G12S & $\begin{array}{l}\text { p.G13C, p.T58I, p.A146V, } \\
\text { p.G13D }\end{array}$ & $0.38(0.89)$ & 0.678 \\
\hline p.G12S & All other mutation groups & $0.87(0.82)$ & 0.289 \\
\hline p.G12A, p.G12C & $\begin{array}{l}\text { p.G13C, p.T58I, p.A146V, } \\
\text { p.G13D }\end{array}$ & $3.22(1.38)$ & 0.027 \\
\hline p.G12A, p.G12C & All other mutation groups & $2.90(1.01)$ & 0.007 \\
\hline
\end{tabular}

$P$ values shown in bold are statistically significant.

and comparing them with the p.G12S mutation group $(P=$ 0.009 ). When the group with p.G12A or p.G12C was compared with the subgroup with mutations other than p.G12S, a significant difference was again found $(P=0.027)$. Finally, the group of individuals with p.G12A or p.G12C was compared with a group containing all subjects with an HRAS mutation other than p.G12A or p.G12C, and a significant difference was found $(P=0.007)$ (Table 5).

\section{DISCUSSION}

\section{Costello syndrome severity over time}

No significant increase in the severity score occurred over time (Supplementary Table S1 online). Although new characteristics arose, they did not arise at a significant rate. This relatively stable severity score suggests that if an individual was moderately affected early in life, he or she remained moderately affected in childhood and young adulthood, as measured by this severity score. There were no periods of an individual's life in which there was a dramatic increase in Costello syndrome severity. On average, a person gained approximately one to two points (or presented with one to two additional characteristics of Costello syndrome) between 2 and 20 years. This quantitative assessment is consistent with the anecdotal experience of many severe medical issues, including the feeding difficulties, cardiac rhythm anomalies, and cerebellar tonsillar herniation manifesting in the first 2 years of life. Although malignancies may be diagnosed after the age of 2 years, these too occur more often in younger individuals, as reviewed by Kratz et al. ${ }^{14}$

\section{Mortality}

Five of the 78 individuals in this study were deceased. Of note, all five individuals received points for use of a feeding tube and four of five scored points for arrhythmia. However, although all five deceased participants had used a feeding tube, 57 other individuals who required a feeding tube are alive. Similarly, 32 individuals with a history of arrhythmia are alive. More data are needed to identify the factors associated with mortality in Costello syndrome. It is noteworthy that while three of the five deceased individuals shared the common mutation predicting p.G12S, two had the mutation predicting G12A or p.G12C, the two mutations shown here to be associated with higher severity scores and thus a more severe phenotype.

\section{Early childhood severity}

A significant difference was found when the mean of the early childhood severity scores of individuals with the p.G12S mutation was compared with the mean of the early childhood severity scores of individuals with p.G12A or p.G12C, indicating a lesser phenotypic severity associated with the HRAS mutation p.G12S as compared with p.G12A and p.G12C. When the p.G12A and p.G12C mutation groups were individually compared with the p.G12S group, the p.G12C group differed more from the p.G12S group than the p.G12A group, indicating a possible spectrum of severity with p.G12C associated with the most severe phenotype, followed closely by the p.G12A mutation. However, due to the small number of individuals with these rare changes, more information is needed to confirm this.

There was no significant difference between the scores of the p.G12S mutation group and the p.G13C $(P=0.87)$ or p.T58I $(P=0.36)$ groups. By contrast, a cohort of 12 individuals with the p.G13C HRAS mutation was recently characterized and 
compared with individuals with the p.G12S HRAS mutation..$^{13}$ In that study, those with p.G13C had significantly lower rates of neurological abnormalities requiring surgery, lack of multifocal atrial tachycardia and papillomata, and a higher likelihood of dolichocilia, or extremely long eyelashes requiring trimming. The differing results of comparisons between those with p.G13C and p.G12S in these two studies may be due to the larger number of individuals studied in Gripp et al. ${ }^{13}$ not all of these individuals were included in the current study. Furthermore, Gripp et al. ${ }^{13}$ focused solely on the phenotypic description of individuals with p.G13C and did not use a standardized approach as applied here. Similarly, an individual with the p.T58I HRAS mutation was reported to have milder facial findings but a comparable severity of Costello syndrome in other respects. ${ }^{12}$ This study reports similar findings, given that this scoring system did not take facial features into consideration.

\section{Limitations of this study}

Major limitations of this study include the relatively small number of individuals with the rarer Costello syndrome-causing HRAS mutations and the parent-reported information with the lack of complete medical record documentation. Although every effort was made to confirm findings with medical records, these records were not always available, and parental report was sometimes used to notate the symptoms present in an individual. If the parents or caregivers of the individual incorrectly reported a symptom that was subsequently scored, this score would be invalid. Some individuals were lost to follow-up, so it was unknown if they developed new findings since their records were last updated. This inconsistency could lead to falsely low scores for those individuals for whom less information was available.

Due to the rare nature of Costello syndrome, only 78 individuals were available for scoring (Table 1 ), and this small sample size limits the statistical significance of our results. Of the 78 individuals, 60 had the p.G12S HRAS change. The finding that the p.G12A and p.G12C changes were associated with more severe phenotypes was based on the groups containing four and three subjects, respectively. The small sample sizes of these mutation groups must be taken into account when generalizing findings to the Costello syndrome population.

\section{Conclusion}

Our data support a correlation between the specific Costello syndrome-causing HRAS change and severity of medical problems in the affected individual. It is conceivable that such information will be used in the future for medical decision making. Unfortunately, no individuals with the rare p.G12V mutation were enrolled in our study, and therefore we cannot provide quantification of its anecdotally severe and typically neonatal lethal phenotype. Although an improved understanding of the implication of the specific mutation could be meaningful to the affected families, a validated severity scoring system may be even more important for future medication trials in this patient population. Due to its defining HRAS mutations, Costello syndrome is considered a model disorder for other RASopathies.
Treatment aimed at alleviating the numerous manifestations of these conditions will likely focus first on Costello syndrome. ${ }^{15}$ Although the severity scoring system described here cannot measure the short-term effects of drug treatment, it is useful in quantifying the differences in severity anticipated with the respective mutations and may thereby guide enrollment criteria. Furthermore, changes in the long-term outcome may be assessed through this severity score. Clearly, further studies are needed to validate and expand the data presented here.

\section{SUPPLEMENTARY MATERIAL}

Supplementary material is linked to the online version of the paper at http://www.nature.com/gim

\section{ACKNOWLEDGMENTS}

We thank the individuals with Costello syndrome and their families for participating in research. We also thank the Arcadia University Genetic Counseling Program and the Ellington Beavers Award for Intellectual Inquiry, which supported this research.

\section{DISCLOSURE}

The authors declare no conflict of interest.

\section{REFERENCES}

1. Lin AE, Alexander ME, Colan SD, et al. Clinical, pathological, and molecular analyses of cardiovascular abnormalities in Costello syndrome: a Ras/MAPK pathway syndrome. Am J Med Genet A 2011;155A:486-507.

2. Axelrad ME, Schwartz DD, Katzenstein JM, Hopkins E, Gripp KW Neurocognitive, adaptive, and behavioral functioning of individuals with Costello syndrome: a review. Am J Med Genet C Semin Med Genet 2011;157:115-122.

3. Gripp KW, Hopkins E, Doyle D, Dobyns WB. High incidence of progressive postnatal cerebellar enlargement in Costello syndrome: brain overgrowth associated with HRAS mutations as the likely cause of structural brain and spinal cord abnormalities. Am J Med Genet A 2010;152A:1161-1168.

4. Gripp KW. Tumor predisposition in Costello syndrome. Am J Med Genet C Semin Med Genet 2005;137C:72-77.

5. Aoki Y, Niihori T, Kawame H, et al. Germline mutations in HRAS proto-oncogene cause Costello syndrome. Nat Genet 2005;37:1038-1040.

6. Estep AL, Tidyman WE, Teitell MA, Cotter PD, Rauen KA. HRAS mutations in Costello syndrome: detection of constitutional activating mutations in codon 12 and 13 and loss of wild-type allele in malignancy. Am J Med Genet A 2006;140:8-16.

7. Gripp KW, Lin AE, Stabley DL, et al. HRAS mutation analysis in Costello syndrome: genotype and phenotype correlation. Am J Med Genet A 2006;140:1-7.

8. Kerr B, Delrue MA, Sigaudy S, et al. Genotype-phenotype correlation in Costello syndrome: HRAS mutation analysis in 43 cases. J Med Genet 2006;43:401-405.

9. Zampino G, Pantaleoni F, Carta C, et al. Diversity, parental germline origin, and phenotypic spectrum of de novo HRAS missense changes in Costello syndrome. Hum Mutat 2007;28:265-272.

10. Gripp KW, Lin AE. Costello syndrome: A Ras/mitogen activated protein kinase pathway syndrome (rasopathy) resulting from HRAS germline mutations. Genet Med 2012;14:285-292.

11. Lo IF, Brewer C, Shannon N, et al. Severe neonatal manifestations of Costello syndrome. J Med Genet 2008;45:167-171.

12. Gripp KW, Innes AM, Axelrad ME, et al. Costello syndrome associated with novel germline HRAS mutations: an attenuated phenotype? Am J Med Genet $A$ 2008;146A:683-690.

13. Gripp KW, Hopkins E, Sol-Church K, et al. Phenotypic analysis of individuals with Costello syndrome due to HRAS p.G13C. Am J Med Genet A 2011;155A:706716.

14. Kratz CP, Rapisuwon S, Reed H, Hasle H, Rosenberg PS. Cancer in Noonan, Costello, cardiofaciocutaneous and LEOPARD syndromes. Am J Med Genet C Semin Med Genet 2011;157:83-89.

15. Rauen KA, Schoyer L, McCormick F, et al. Proceedings from the 2009 genetic syndromes of the Ras/MAPK pathway: from bedside to bench and back. Am J Med Genet A 2010;152A:4-24. 